\title{
NEURONAL ACTIVITY IN THE ISOLATED CORTICAL SLABS OF THE CAT*
}

\author{
Satoru Watanabe AND Hiromi Yuasa** \\ Department of Pinysiology, Gifu University School of Medicine, Gifu
}

The activity of the sensory cortex is subjected to thalamic control even in the absence of external stimulation. The rhythmic electrical waves of the cerebral cortex are largely due to the thalamo-cortical reverberating relationship, as suggested by ECCLES (1963). Interspike intervals of spontaneous discharges show different distributions in different parts of the visual pathway ; the exponential distribution in the optic tract, bi- and tri-modal distributions in the lateral geniculate body and a distribution with a peak at short intervals followed by wide and unsystematic scattering of longer intervals in the cortex (HERz et al. 1964). These facts suggest that neurons in the higher centers of the visual system are influenced not only by specific inputs, but also by nonspecific ones of nonvisual systems or afferents. Moreover, there are profusely complexed connections of intracortical association or axon collaterals among cortical neurons (SHOLL, 1956).

In order to eliminate the influences of thalamic or afferent connections coming up the white matter, chronically isolated slabs of the visual cortex were prepared in the experiment reported here. A microelectrode study of the chronically deafferentiated preparations of the visual cortex was first described by CREUTzFELDT and STRUCK (1962). They did not observe any obvious rhythmic spontaneous EEG, but some spontaneous activities of neurons in synchrony with grouped fast EEG waves. They suggested that these activities revealed a pure intracortical "Eigenrhythmus".

In this paper, the spontaneous postsynaptic potentials (PSPs) recorded intracellularly with microelectrodes and responses of neurons produced by epicortical stimulation in isolated cortical slabs were analyzed to study intracortical linkages. Moreover, the question whether intracortical inhibitory

Received for publication August 31, 1970.

渡辺 悟, 湯浅博実

* This study was supported in part by a grant (No. B. 8818) of Ministry of Education of Japan, and includes partly work done in Max-Planck Institute for Psychiatry, Munich, Germany.

** Present address: Department of Neurosurgery, Nagoya University School of Medicine, Nagoya. 
pathways are composed only of forward pathways or also of backward pathways, will be examined.

\section{METHODS}

Cats were anesthetized with Nembutal injected intraperitoneally $(30-40 \mathrm{mg} / \mathrm{Kg}$ ). The part of the skull covering the lateral, suprasylvian and ectosylvian gyri was removed. After cutting the dura, circular piece of tissue $3 \mathrm{~mm}$ in thickness and 20 $\mathrm{mm}$ in diameter containing parts of the lateral and suprasylvian gyri was detached by slicing with a thin curved knife from all subcortical as well as intracortical connections except the superficial blood vessels contained in the pia. After the operation the fascia were returned to their original positions to be sewn together, and the cut in the skin was closed by sutures. Fourteen cats were used in this experiment. They were individually caged and used for intracellular studies 4 to 6 weeks after the operation. These cats were again operated for acute experiments. The cerebrospinal fluid was drained through an incision made in the cisterna magma in order to reduce the movement of the brain. In order to prevent the cortical surface from drying a thin layer of agar-agar ( $1 \%$ dissolved in Ringer solution) was placed on it after cleaning the wound and holding the cortex down by a plastic pressure foot $(1.5 \mathrm{~cm}$ in diameter) attached on the stereotaxic instrument. The pressure foot was provided with a $\mathrm{Ag}-\mathrm{AgCl}$ ball electrode for surface recording, two $\mathrm{Ag}-\mathrm{AgCl}$ ball electrodes for epicortical stimulation and a small hole $(1 \mathrm{~mm}$ in diameter) in its center for inserting the microelectrode. Agar-agar under this hole was removed to allow the passage of the electrode.

Surface corticogram as well as intracellular recording was made in the isolated cortical slabs. For intracellular recording glass capillary electrodes filled with $1.5 \mathrm{~mol}$ potassium citrate were used. Electrodes of which the d.c. resistances ranged from 30 to $70 \mathrm{meg}$ ohms were selected for use. The microelectrode was connected to a high input impedance preamplifier. Recording was made by a long recording camera for photographing the shape of electrical signals on a dual beam oscilloscope, and by a data recorder connected to the amplifier output.

Intracellular application of currents through a recording electrode was accomplished with a balancing method of Wheatstone bridge containing a fixed resistance of 1 giga ohms. Stimulating currents were supplied from a square pulse generator. The same stimulator was used for the epicortical stimulation through a pair of silver ball electrodes.

Histological examination was performed in order to confirmed whether the cortical preparation was isolated completely from other tissues.

\section{RESULTS}

\section{Spontaneous postsynaptic potentials}

The resting membrane potentials of neurons in the isolated preparations as measured immediately after electrode penetration were lower in mean value of $-30 \mathrm{mV}$ and standard deviation of $\pm 7.0 \mathrm{mV}$ than those obtained in an intact visual cortex. According to an experiment on degenerated geniculate neurons ( $\mathrm{KocH}$ et al. 1962), a transneuronally degenerated neuron exhibited little change in cellular ionic compositions with slightly elevated $\mathrm{Na}^{+}$and $\mathrm{K}^{+}$, and lowered $\mathrm{Cl}^{-}$. On the other hand, a retrogradely degenerated neuron 
showed marked changes with much higher concentrations of $\mathrm{Na}^{+}$and $\mathrm{Cl}^{-}$, and a slightly lower concentration of $\mathrm{K}^{+}$. The former could be only slightly depolarized and the latter could no longer excite itself. Therefore, the impaled neurons which possessed a resting potential of about $-30 \mathrm{mV}$ might be normal or transneuronally degenerated. After injury discharges disappeared, the membrane potential held a relatively stable level for more than 10 minutes (FIG. 1). In preparations under Nembutal anesthesia the spontaneous activity

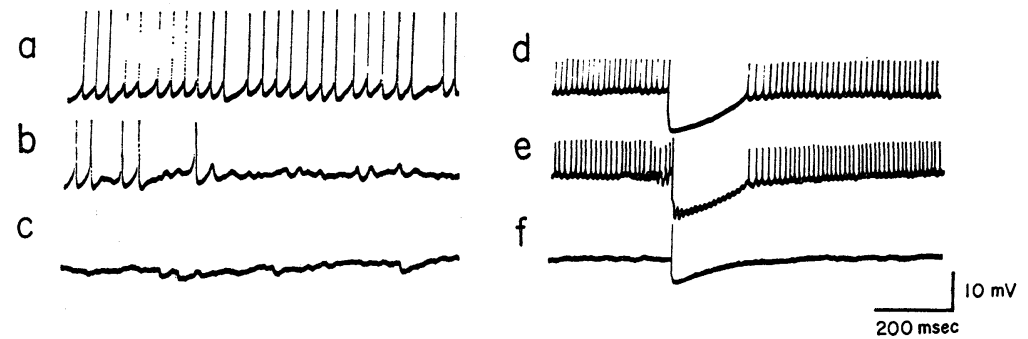

FIG. 1. Spontaneous activity of a neuron in chronically isolated cortex. a : immediately after the penetration of the electrode, the cell fired injury discharges. b: after injury discharge the membrane potential oscillated spontaneously. c: spontaneous IPSPs. $d$, e and $f$ : long lasting IPSPs produced by epicortical stimulation. $d$ and e are during injury discharges, especially $\mathrm{e}$ is oscillatory potentials. $\mathrm{f}$ : an evoked long lasting IPSP after the cessation of injury discharges.

of the neuron was seldom found, also flat EEG was seen in the slabs. Some neurons showed a periodic activity with a long interval of $6-10 \mathrm{sec}$, as described by CREUTzFELDT and STRUCK. On the other hand, a considerable number of spontaneous PSPs were recorded from the slabs, when the animals were not anesthetized but just immobilized with gallamine triiodethylate (FIG. 1c). The amplitude of spontaneous PSPs was smaller than that of motoneurons of the cat (Kuno, 1964) and the frog (KATZ and MiLEd, 1963) and ciliary ganglion cells of the chick (MARTINE and PILAR, 1964), but there was no significant difference in the amplitude of PSPs between isolated cortex and intact visual cortex. The observed spontaneous PSPs were not grouped but solitary in nature indicating that they are due to random synaptic noise rather than to afferent bombardments. These facts suggest that the spontaneous PSPs smaller than $1 \mathrm{mV}$ might be true miniature PSPs similar to the miniature endplate potential. FIG. 2A shows an amplitude histogram with mean values of $0.9 \mathrm{mV}$ for spontaneous EPSPs and $0.8 \mathrm{mV}$ for the spontaneous IPSPs, comprising 245 EPSPs and 62 IPSPs obtained in a period of $10 \mathrm{sec}$. Spontaneous IPSPs in isolated slabs appeared more frequently, about five to ten folds, than in the intact cortex. The interval histogram of spontaneous PSPs showed an exponential or a Poisson distribution (FIG. 2B). Spontaneous PSPs occur less 

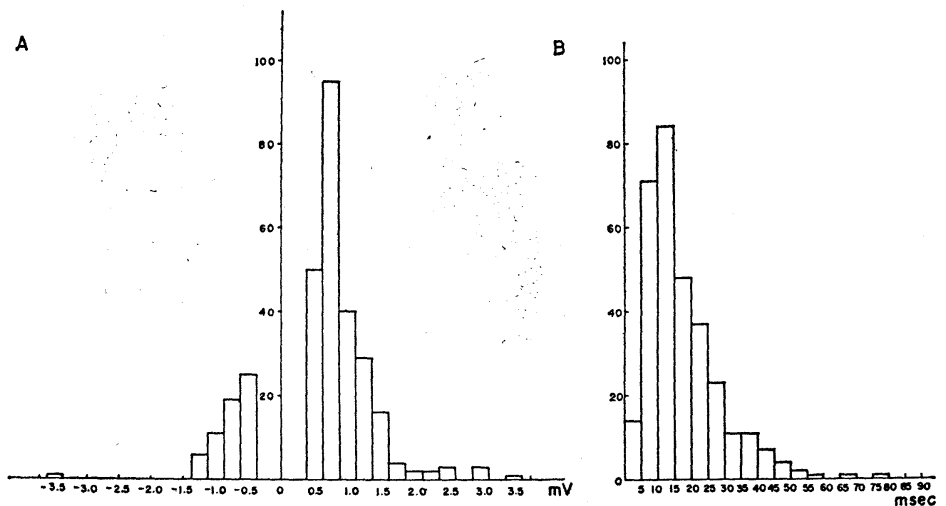

FIG. 2. Amplitude histogram (A) and interval histogram (B) of spontaneous PSPs recorded from an isolated cortical slab. A and B are measured on the same cell and graphs are based on different $10 \mathrm{sec}$ samples. Ordinates of $A$ and $B$ are frequency of PSPs. Abscissa of A is amplitude of PSP and B is interval between PSPs.

frequently ( $40 \%$ of intact cortex) in the isolated cortex than in the intact one, as estimated by random sampling of $10 \mathrm{sec}$ intervals.

2. Effects of tetrodotoxin on the synaptic noise of cortical neurons

Tetrodotoxin is known to block conduction of nerve impulses without interfering with spontaneous release of transmitters and the postsynaptic effects of transmitters. It would be interesting to compare the effects of tetrodotoxin with those by the surgical isolation method. Tetrodotoxin $(0.4$

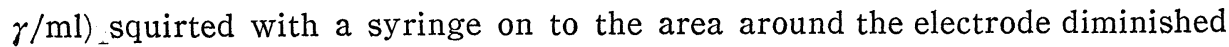

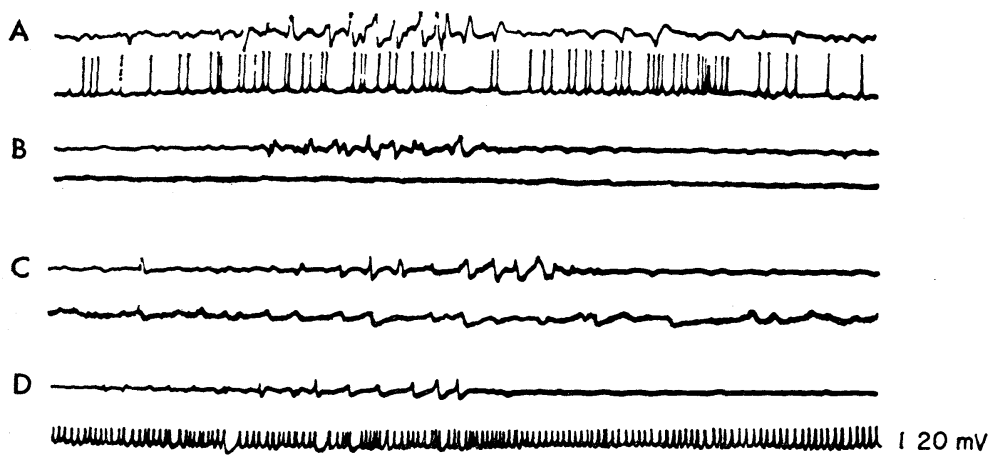

$\overline{400 \mathrm{msec}}$

FIG. 3. Effects of tetrodotoxin on spontaneous PSPs of an intact visual cortical neuron. A: before tetrodotoxin application. B: after 11 minutes. C: after 20 minutes. D : after 30 minutes of tetrodotoxin application. Upper traces: EEG. Lower traces: intracellular recording. 
the spontaneous PSPs eleven minutes later, and then the membrane potential of the impaled cell was greatly depolarized above $10 \mathrm{mV}$ (FIG. 3). At that time the spindle waves of EEG were not yet affected. After 20 minutes, accompanying the recovery of membrane depolarization, occassionally small hyperpolarizing potentials appeared. The flattening of EEG was delayed until about 30 minutes after the application of tetrodotoxin when spike discharge already began to reappear. As confirmed by HubBard, STENHAUSEN and ROSAMOND ECCLES (1967) in the motor cortex of the kitten, some true miniature PSPs undoubtedly remain after tetrodotoxin application, but most synaptic noise due to transmitter release caused by nerve impulses can be removed by blocking of nerve impulses. The intact cortex seems to be activated mostly by impulses from the afferent axons and their collaterals including the intracortical connections.

\section{Epicortical stimulation on the slabs}

Direct stimulation of a chronically isolated cortical slab produced a direct cortical response (GOLDRING et al., 1961) and simultaneously a long lasting hyperpolarization as in the intact cortex. FIG. If and FIG. 5 show a hyperpolarizing potential by epicortical stimulation after cessation of injury discharges. During injury discharges (FIG. 1d and e) the membrane potential revealed oscillatory fluctuations with spikes corresponding to the oscillatory frequency. Also the oscillatory fluctuations appeared even during hyperpolarization evoked by epicortical stimulation (FIG. 1e). The hyperpolarizing potential was occassionally preceded by a spike potential, as shown in Fig. 5 . The hyperpolarizing potential can be made to vanish by applying appropriate

A

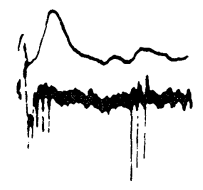

C

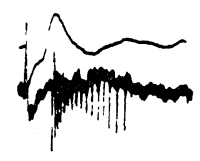

B
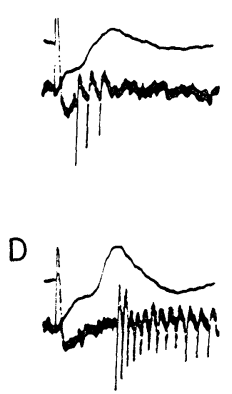

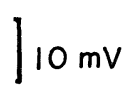

$\overline{80 \mathrm{msec}}$

$40 \mathrm{msec}$

FIG. 4. Interneuronal discharge patterns in a chronically isolated cortical slab. The same neuron showed two different modes of responses: discharge and pause, respectively $A$ to $B$ and $C$ to $D$. Upper traces: EEG. Lower traces: extracellular recording. 


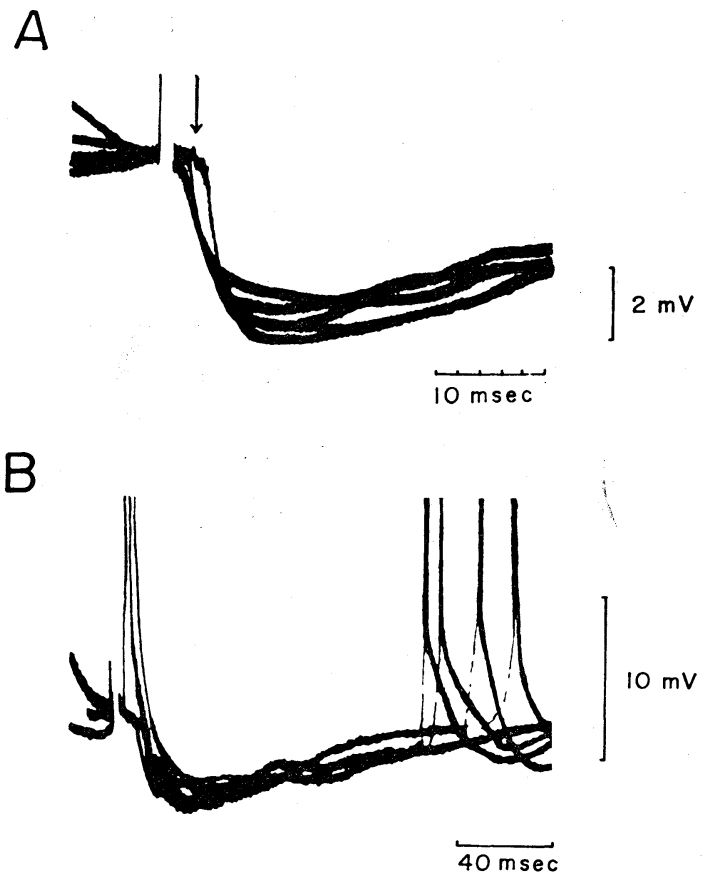

FIG. 5. A long lasting IPSP produced by epicortical stimulation on an isolated cortex. A : without spike and B: preceded by an antidromic spike. Arrow shows the initiation of IPSP.

anodal currents through the recording electrode, as commonly seen with IPSPs (FIG. 6). The mean latency of IPSPs after the onset of stimulation was 2.8 msec depending on the stimulus intensities. The latency did not vary so much whether a spike potential preceded or not. This fact shows that this long lasting IPSP should be produced bi-synaptically via a inhibitory interneuron. If the interneuron was invaded directly by electrical shock, the IPSP should be evoked mono-synaptically on the pyramidal cell. As shown in FIG. 5, no IPSP appeared earlier than $1.7 \mathrm{msec}$. This bisynaptical sign reveals the existence of an inhibitory interneuron presumably connected with the recurrent collaterals of pyramidal cells, since in the isolated slab there is no afferent fiber coming up from remote sites.

In some cases, grouping discharge patterns evoked by epicortical stimulation were recorded extracellularly in the slabs. FIG. 4 shows the responses of a neuron, consisting of an initial burst discharge with a frequency of about 150 spikes per sec during first $30-40 \mathrm{msec}$ and a pause of $80-100 \mathrm{msec}$ followed by a burst discharge. The initial burst corresponded to the rising phase of the negativity of surface potentials. Furthermore, in some cases the same cell that responded in the above manner released a burst of impulses $80-100$ 
msec in length during the pause period mentioned above. Thus, two different firing patterns were observed in a cell as if it was controlled by some reciprocal process like a flip-flop.

\section{Effects of intracellularly applied currents}

When the membrane was depolarized by cathodal currents through the recording electrode connected to a bridge circuit, the long lasting hyperpolarizing potential produced by direct stimulation was enhanced by increasing currents, as shown in FIG. 6. Concurrently, the depolarizing potential which was superimposed on the hyperpolarizing potential, became smaller. Applying anodal currents, the hyperpolarizing potential became invisible by further increasing currents, and the superimposed depolarizing potential became larger. The effects of intracellularly applied currents on the long lasting hyperpolarizing potential and on the superimposed depolarizing potential indicate the identity of these potentials respectively as IPSP and EPSP. These synaptic potentials, both IPSP and EPSP might also be produced near the recording site in the soma, since both synaptic potentials changed linearly with the applied currents (FIG. 7). Concerning with synaptic sites of neurons,

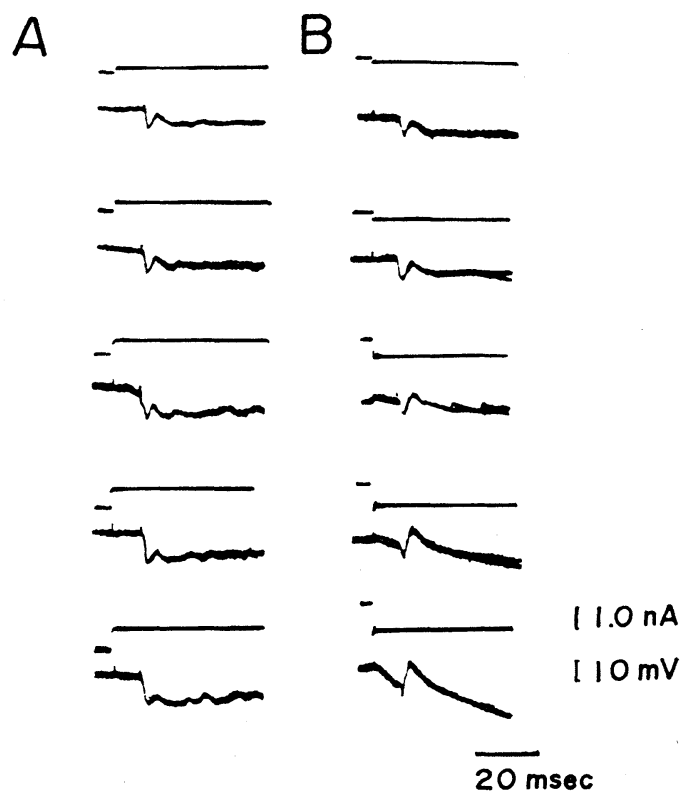

FIG. 6. Effects of applied injected currents intracellularly on IPSP and EPSP of a neuron in chronically isolated cortex. Left column shows effects of depolarizing currents and right column hyperpolarizing currents, which enhanced an unmasked EPSP superimposed on IPSP. Upper traces represent the polarizing currents, depolarizing upward and hyperpolarizing downward. 

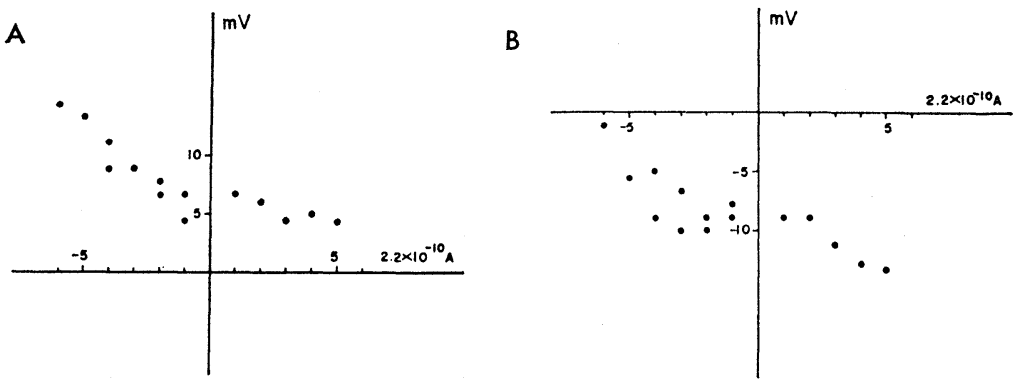

FIG. 7. Scatter diagrams plotting the amplitude of EPSPs (A) and IPSPs (B) against the intracellularly applied currents. See text.

our findings agree well with those of electron microscopic studies (CoLONNIER, 1966), which showed only axosomatic synapses in chronically isolated cortical slabs.

\section{DISCUSSION}

1. Synaptic noise in isolated cortical slabs

In the pericruciate areas of the cat, the spontaneous PSPs of the cortical cells are composed of "unitary" EPSPs and IPSPs due to afferent and collateral fiber activities and not of true miniature potentials due to spontaneous liberation of transmitter substances, as reported in our previous paper (1966). The previous experiments were performed under Nembutal anesthesia. Neurons in the chronically isolated cortical slabs, deafferentiated preparations, did not reveal any spontaneous PSPs, but reacted with spikes and long lasting IPSPs to epicortical stimulation. It is difficult to determine whether Nembutal affects the fiber terminals or not. However, Nembutal does not change the excitability of cortical neurons, but might depress the synapses near the initial segment of neurons (KITASATO, 1965). In the cortical slabs, under immobilized conditions with gallamine, spontaneous PSPs were observed as in intact cortical neurons. Thus Nembutal might block the activities of interneurons. Interval and amplitude histograms of spontaneous PSPs showed Poisson distributions. The spontaneous PSPs appeared with a random process represent the unitary PSPs. Their random property means synaptic noise, and might be attributed to the activity of the intracortical connections, which does not exhibit any regular rhythmicity except for a periodic grouped discharge with 6-10 sec intervals. Application of tetrodotoxin on the intact visual cortex caused a decrease in the frequency of spontaneous PSPs, but did not affect that of spontaneous IPSPs. The depolarization of membrane potential by tetrodotoxin caused the diminution of spontaneous EPSPs and simultaneously the enhancement of spontaneous IPSPs. Even if tetrodotoxin 
does not interfer with the spontaneous release of transmitter substances or the postsynaptic effects of transmitter substances, it is hard to conclude so easily that the synaptic noise in the cortical neurons is not necessarily evoked only by nerve impulses. As the potential of postsynaptic membrane was depolarized by application of tetrodotoxin, depolarizing PSPs might be masked. There are undoubtedly some spontaneous PSPs similar to the miniature endplate potentials of peripheral synapses. The spontaneous PSPs recorded from the isolated cortical slabs or under tetrodotoxin application, are smaller in amplitude and lower in frequency than those in the intact cortex, and formed mostly "solitary" EPSPs. The spontaneous PSPs which formed groups or cascaded simultaneously with the spindle burst activity, were seldom found in the isolated cortical slabs. The synaptic noise observed in isolated cortical slabs appears to consist of PSPs produced in pyramidal cells by impulses of intracortical linkages including collaterals and of spontaneous miniature PSPs.

\section{Forward and backward inhibitory pathways in the cortical layers}

In the visual cortex, including the striate and parastriate cortex, a long lasting IPSP was evoked by stimulation of various parts of the visual pathways. As pointed out earlier (1966), the latency of IPSPs was longer than that of EPSPs, and the intervals between the onset of IPSP and that of EPSP elicited by stimulation of the optic tract was calculated to be $1.0 \mathrm{msec}$ for " fast group" and 0.9 msec for "slow group". Recently, Toy AMA et al. have reported that the latency of the IPSPs evoked by stimulation of the optic chiasma, lateral geniculate body and optic radiation was also regularly longer by 0.8 msec than that of the EPSP. This difference of $0.1-0.2 \mathrm{msec}$ does not suffice to assume that the IPSPs might be produced at least by an interneuron located between two pyramidal cells. Provided that the specific visual afferents were stimulated, the forward inhibitory pathway, as described by EcCLES (1965), might be the possible interpretation. However, stimulation of the optic radiation elicits an antidromic spike followed by a long lasting IPSP. In the visual cortex, three types of efferent fibers were known physiologically, as association efferents, commissural efferents and corticofugal efferents. A histological classification of pyramidal cells in cerebral cortex by SHOLL (1956) divided them into four types, in which three types (P1, P2 and P3) extend an axon down to the white matter, and two types (P3 and P4) possess recurrent collaterals. Stimulation of these efferent fibers could generate antidromic spikes in pyramidal cells, and accompanying long lasting IPSPs which are produced bisynaptically through the recurrent collaterals of the pyramidal cells. However, this method of antidromic volley is insufficient to use as evidence for recurrent collaterals, because the optic radiation and commissural tract contain not only efferent, but also afferent fibers. No afferent fibers: 
coming from the white matter survive in the slabs 4-6 weeks after operation. COLONNIER has demonstrated that afferent terminals in a chronically isolated slab were phagocyted by glial cells. There were only axosomatic synapses, that occur in intracortical neurons remained. He suggested, moreover, that afferent fibers to the cortex synapsed mainly on dendritic spines and trunks with axodendritic synapses, and that a large number of intracortical fibers made contact cell bodies and dendritic trunks as axosomatic synapses. In chronically isolated cortical slabs, a bisynaptically evoked IPSP reveals the existence of backward inhibitory pathways.

Presumably the bisynaptic IPSP in the chronically isolated cortical slab is produced by an inhibitory interneuron connected with the recurrent collaterals of the pyramidal cell. These backward inhibitory pathways play an important role in the functional columnar organization of the visual cortex, as described by HUBEL and WIESEL (1963). Ascending afferent fibers running parallel to the long axis of pyramidal cells may be mainly excitatory. Recurrent collaterals of these cells may inhibit adjoining pyramidal cells via interneurons or stellate cells, and modify not only excitatory but also forward inhibitory actions.

\section{SUMMARY}

1. In isolated cortical slabs, intracellular recording was performed. The average membrane potential of impaled cells was $-30 \mathrm{mV}$ with a S. D. of $\pm 7.0 \mathrm{mV}$. In these cells there were spontaneous postsynaptic potentials or synaptic noise, which were mostly solitary PSPs. The frequency of PSPs amounted to about $40 \%$ of that of the intact visual cortex.

2. Spontaneous IPSPs appeared more frequently in an isolated slab than in an intact cortex. Inhibitory as well as excitatory interneurons were found in isolated cortical slabs.

3. By application of tetrodotoxin in an intact cortex, the spontaneous PSPS disappeared within first 20 minutes, while in this period the membrane potential was largely depolarized.

4. Epicortical stimulation of an isolated cortex produces a long lasting IPSP as in an intact cortex. The mean latency of IPSPs was $2.8 \mathrm{msec}$ with or without a preceding spike. No IPSP was elicited earlier than $1.7 \mathrm{msec}$. It was concluded that the intracortically produced IPSP was caused bi-synaptically by an inhibitory interneuron which might be connected with recurrent collaterals of cortical pyramidal cells.

The authors would like to express gratitude to Dr. M. Konishi, Princeton Uniwersity, for the correction of the manuscript. 


\section{REFERENCES}

1) Colonnier, M.L.: Experimental degeneration in the cerebral cortex. J. Anat., $98: 47-53,1964$.

2) Colonnier, M.L.: The structural design of the neocortex. in "Brain and conscious experience" Ed. J.C. ECCLES, p. 1-23, 1966.

3) Creutzfeldt, O.D. And Struck, G.: Neurophysiologie und Morphologie der chronisch isolierten Cortexinsel der Katze: Hirnpotentiale und Neurontatigkeit einer isolierten Nervenzellpopulation ohne afferente Fasern. Arch. Psychiat. Ner. venkr., 203 : 708-731, 1962.

4) ECCLES, J.C.: The physiology of synapses. Springer-Verl. 1964.

5) Goldring, S., O'Leary, J.L., Helmes, T.G. and Jerva, M.J.: Direct responses of isolated cerebral cortex of cat. J. Neurophysiol., 24 : 633-650, 1961.

6) Herz, A., Creutzfeldt, O.D. and Fuster, J.M.: Statistische Eigenschaften. der Neuronaktivitat im ascendierenden visuellen System. Kybernetik, 2:61-71, 1964.

7) Hubbard, J.I., Stenhouse, D. and Rosamond M. Eccles: Origin of synaptic noise. Science, 157:330-331, 1967.

8) Hubel, D.H. and Wiesel, T.N.: Shape and arrangement of columns in cat's striate cortex. J. Physiol., 165: 559-568, 1963.

9) Katz, B. ANd Miledi, R.: A study of spontaneous miniature potentials in spinal motoneurons. J. Physiol., $168:$ 389-422, 1963.

10) Kitasato, H.: The change in the surface negative cortical response during. nembutal anesthesia and firing level of neurons in the cortex of rabbit. Jap. J. Physiol., 15: 71-91, 1965.

11) Koch, A., Ranck, J.B. And Newman, B.L.: Ionic content of the neuroglia. Exp. Neurol., 6 : 186-200, 1962.

12) KUno, M.: Quantal components of excitatory synaptic potentials in spinal motoneurons. J. Physiol., 175: 81-99, 1964.

13) Martin, A.R. And Pilar, G.: Quantal components of the synaptic potential in the ciliary ganglion of the chick. J. Physiol., 175:1-16, 1964.

14) SHoll, D.A.: Dendritic organization in the neurons of the visual and motor cortices of the cat. J. Anat., 87: 287-406, 1953.

15) Sholl, D.A.: The organization of the cerebral cortex. London: Methuen, 1956.

16) Stefanis, C. And Jasper, H.: Recurrent collateral inhibition in pyramidal tract neurons. J. Neurophysiol., $27: 855-877,1964$.

17) Toyama, K. and Matsunami, K.: Synaptic action of specific visual impulses upon cat's parastriate cortex. Brain Res., 10:473-476, 1968.

18) Toyama, K., Matsunami, K. and Ohno, T.: Antidromic identification of association, commissural and corticofugal efferent cells in the cat visual cortex. Brain Res., $14:$ 513-517, 1969.

19) Toyama, K., Tokashiki, S. And Matsunami, K.: Synaptic action of commissural impulses upou association efferent cells in cat visual cortex. Brain Res., $14: 518-520,1969$.

20) Watanabe, S. and Creutzfeldt, O.D.: Spontane postsynaptische Potentiale von Nervenzellen des motorischen Cortex der Katze. Exp. Brain Res., 1: 48-64, 1966.

21) Watanabe, S., Konishi, M. and Creutzfeldt, O.D.: Postsynaptic potentials. in the cat's visual cortex following electrical stimulation of afferent pathways. Exp. Brain Res., 1: 272-283, 1966. 